\title{
Variability and predictors of urinary concentrations of organophosphate flame retardant metabolites among pregnant women in Rhode Island
}

Megan E. Romano ${ }^{1,2^{*}}$, Nicola L. Hawley ${ }^{3}$, Melissa Eliot ${ }^{1}$, Antonia M. Calafat ${ }^{4}$, Nayana K. Jayatilaka ${ }^{4}$, Karl Kelsey ${ }^{1,5}$, Stephen McGarvey ${ }^{1,6}$, Maureen G. Phipps ${ }^{1,7}$, David A. Savitz ${ }^{1,7}$, Erika F. Werner ${ }^{7}$ and Joseph M. Braun ${ }^{1}$

\begin{abstract}
Background: Organophospate flame retardants (PFRs) are chemicals of emerging concern due to restrictions on polybrominated diphenyl ether flame retardant formulations. We describe the occurrence, variability, and predictors of urinary metabolites of PFRs among pregnant women.

Methods: In 2014-2015, 59 women from Providence, Rl provided up to 3 spot urine samples during pregnancy $(\sim 12,28$, and 35 weeks' gestation). We created a pooled urine sample per woman and measured nine relevant metabolites in individual and pooled samples. We used linear mixed models to calculate intraclass correlation coefficients (ICCS) across the 3 measurements and to assess sociodemographic and dietary predictors of PFRs.

Results: The median (IQR) of bis-2-chloroethyl phosphate (BCEP), bis(1,3-dichloro-2-propyl) phosphate (BDCPP), and diphenyl phosphate (DPhP), the metabolites most frequently detected, from pooled samples were: $0.31 \mu \mathrm{g} / \mathrm{L}$ $(0.17-0.54), 1.18 \mu \mathrm{g} / \mathrm{L}(0.64-2.19), 0.93 \mu \mathrm{g} / \mathrm{L}(0.72-1.97)$, respectively. We observed fair to good reproducibility for BCEP $(I C C=0.50), B D C P P(I C C=0.60)$, and DPhP $(I C C=0.43)$, and excellent agreement between the urinary flame retardant metabolite concentrations averaged across pregnancy versus pooled urine sample concentrations for BCEP (ICC = 0.95), BDCPP (ICC = 0.89), and DPhP (ICC = 0.93). Adjusting for pertinent sociodemographic factors and gestational week of urine collection, each $1 \mathrm{~kg}$ increase in pre-pregnancy weight was associated with greater $\operatorname{BCEP}(1.1 \% ; 95 \%$ Cl: $0.1,2.1), \operatorname{BDCPP}(1.5 \% ; 95 \% \mathrm{Cl}: 0.3,2.7)$, and DPhP $(0.5 \% ; 95 \%$ Cl: $0.0,1.1)$. Dietary factors were generally not associated with urinary flame retardant metabolites.

Conclusions: Urinary concentrations of BCEP, BDCPP, and DPhP were frequently detected among women in this pilot study and had fair reproducibility across pregnancy. Body size may be an important predictor of urinary flame retardant metabolite concentrations.
\end{abstract}

Keywords: Flame retardants, Pregnancy, Urine metabolites

\footnotetext{
* Correspondence: megan.eromano@dartmouth.edu

'Department of Epidemiology, Brown University School of Public Health,

Providence, RI, USA

${ }^{2}$ Department of Epidemiology, Geisel School of Medicine at Dartmouth,

Lebanon, NH, USA

Full list of author information is available at the end of the article
} 


\section{Background}

To meet state and federal flammability standards, consumer products such as electronics and furniture are often treated with chemical flame retardants [1]. Following the 2004 phase out of specific commercial mixtures of polybrominated diphenyl ether (PBDE) flame retardants due to health and safety concerns, organophosphate flame retardants (PFRs) including triphenyl phosphate (TPhP), tris (1,3-dichloro-2-propyl) phosphate (TDCPP), tris (1-chloro-2-propyl) phosphate, tris (2-chloroethyl) phosphate (TCEP), tri-p- and tri-ocresylphosphate, tributyl phosphate (TBuP), tribenzyl phosphate, and novel brominated flame retardants such as 2-ethylhexyl-2,3,4,5-tetrabromobenzoate, have been increasingly used in consumer products including residential furniture and baby products [1-3]. TPhP, TBuP, and tricresylphosphate are also commonly used as plasticizers or lubricants [3-7].

PFRs have been frequently detected in household dust, foam furniture, and other foam products [1, 2, 8-12], and their metabolites have been identified in the urine of members of the general population of the United States (US) and elsewhere [10-18]. However, data on both human exposure to these chemicals and associated potential adverse health outcomes is still limited, particularly during the sensitive window of pregnancy. Preliminary evidence from epidemiological studies supports a role for PFRs in disruption of thyroid hormones [19-21] and sex hormones among men [20], suggesting that PFRs may have adverse influences on endogenous hormones or hormonally mediated endpoints. In addition, TDCPP is a carcinogen included in the State of California's Proposition 65 list of chemicals known to cause cancer [22]. A small number of prior studies have assessed urinary concentrations of PFR metabolites among maternaltoddler pairs [13, 23, 24], infants [12], or pregnant women $[14,25]$. These studies suggest that pregnant women and young children in the US general population are likely to have detectable concentrations of two urinary metabolites of PFRs, bis(1,3-dicholoro-2-propyl) phosphate (BDCPP) and diphenyl phosphate (DPhP) [12-14], and that such metabolites may be variable over the course of pregnancy $[14,25]$. The extent of exposures among mothers and young children remains largely unknown and sociodemographic and dietary predictors of urinary concentrations of PFR metabolites have not been well described. Information about predictors of exposure are necessary to inform efforts to design epidemiological studies of PFR toxicity, prevent future exposure, and target appropriate safety information.

We conducted a pilot study among pregnant women in Rhode Island to: 1) characterize the occurrence and concentrations of urinary metabolites of PFRs, 2) evaluate the variability of urinary metabolites of these chemicals over the course of pregnancy and explore the utility of pooled urine samples for future research, and 3) investigate associations of these urinary metabolites with sociodemographic and dietary predictors.

\section{Methods}

\section{Study setting and population}

Between July and December 2014, we enrolled 62 women from prenatal clinics affiliated with Women \& Infants Hospital of Rhode Island (WIHRI), which provides care for $75 \%$ of deliveries to state residents. Women were eligible for enrollment if they were $\geq 18$ years old, $\leq 20$ weeks gestation, English speaking residents of Rhode Island, and intended to deliver at WIHRI. Women were excluded if they had a multifetal pregnancy or had been diagnosed with or were currently receiving treatment for serious chronic health issues including, thyroid/renal disorders, HIV, cardiovascular disease other than hypertension, cancer, drug/alcohol addiction, or pre-gestational diabetes. Three women withdrew from the study, leaving 59 women for the present analysis. All women provided written informed consent prior to engaging in study activities and all protocols were approved by the WIHRI institutional review board. The involvement of the Centers for Disease Control and Prevention (CDC) laboratory did not constitute engagement in human subjects research.

\section{Urine sample collection and quantification of urinary flame retardant metabolite concentrations}

We collected spot urine samples in polypropylene specimen cups during clinic visits at three time points during pregnancy: enrollment (12 \pm 2 gestational weeks) and two visits coinciding with routine antenatal gestational diabetes screening ( $28 \pm 2$ gestational weeks) and group B streptococcus (35 \pm 1 gestational weeks) screening tests. All 59 women provided at least one urine sample during pregnancy, 54 (91\%) provided at least two samples, and 41 (70\%) provided all three samples. Urine samples were immediately refrigerated following collection. Urine samples were vortexed for $30 \mathrm{~s}$ and specific gravity (SG) was measured using a handheld digital refractometer (ATAGO, PAS-10S) to quantify urine dilution. Then urine was aliquoted into polypropylene cryovials and stored at $-80{ }^{\circ} \mathrm{C}$ within $24 \mathrm{~h}$ of collection. We also created a pooled urine sample for each woman using $1 \mathrm{~mL}$ of urine from each of her individual samples at the time of preparing the samples for shipment to the Division of Laboratory Sciences, National Center for Environmental Health, CDC (Atlanta, Georgia, USA). All samples were shipped on dry ice to the CDC, where they were stored at or below $-20{ }^{\circ} \mathrm{C}$ until analysis. 
Concentrations of nine urinary flame retardant metabolites, namely DPhP, BDCPP, bis-(1-chloro-2-propyl) phosphate (BCPP), bis-2-chloroethyl phosphate (BCEP), di-p-cresylphosphate (DpCP), di-o-cresylphosphate (DoCP), dibutyl phosphate (DBuP), di-benzyl-phosphate (DBzP), and 2,3,4,5-tetrabromobenzoic acid (TBBA) were quantified in individual and pooled samples [3]. These are urinary metabolites of TPhP, TDCPP, tris (1-chloro-2-propyl) phosphate, TCEP, tri-p-cresylphosphate, tri-o-cresylphosphate, TBuP, tribenzyl phosphate, and 2-ethylhexyl-2,3,4,5-tetrabromobenzoate, respectively. The method uses $0.4 \mathrm{~mL}$ of urine and relies on an enzymatic hydrolysis of urinary conjugates followed by automated off-line solid phase extraction with a polymeric weak anion exchange cartridge to pre-concentrate the target compounds while minimizing potential urine matrix interferences and increasing overall sensitivity and specificity. The deconjugated target analytes in the urine extract are separated on an ultra-highperformance liquid chromatography system with reversed phase chromatography, and quantified by isotope dilutionnegative ion electrospray ionization tandem mass spectrometry. Spiked recoveries at four concentrations of the native analytes $(2,8,16,30 \mathrm{ng} / \mathrm{mL})$ ranged from 90 to $113 \%$, depending on the analyte. Relative standard deviations of repeated analyses of urine spiked with 1, 8 and $20 \mu \mathrm{g} / \mathrm{L}$ of the target analytes were $<10 \%$. The limit of detection (LOD) was estimated from 20 repeated measurements of low concentration standards after plotting the standard deviation of the measured concentration versus the standard concentration. The standard deviation at zero concentration, $\mathrm{S}_{0}$, was determined by the $\mathrm{y}$ intercept of a linear regression analysis of the above plot; LODs were calculated as three times $S_{0}$ [26]. The LODs for the individual metabolites ranged from 0.05 to $0.16 \mu \mathrm{g} / \mathrm{L}$, depending on the analyte (Additional file 1: Table S1). Along with the study samples, each analytical run included high- and lowconcentration quality control materials (QCs) and reagent blanks to assure the accuracy and reliability of the data. The concentrations of the high-concentration QCs and the low-concentration QCs, averaged to obtain one measurement of high-concentration QC and low-concentration QC for each run, were evaluated using standard statistical probability rules [27]. In addition to the internal CDC QC procedures, we incorporated three field blanks (made with laboratory grade water) and 12 masked QC specimens from a single urine pool prepared at Brown University. The coefficients of variation ( $\mathrm{SD} /$ mean concentration) of the blind QCs were $<11 \%$ for the six analytes detected in at least $75 \%$ of samples (Additional file 1: Table S2). Concentrations of all analytes were $<$ LOD in the reagent and field blanks.

Concentrations of urinary metabolites of interest were SG standardized using a modification of a previously described formula: $P_{c}=P\left[S G_{r e f}-1 / S G-1\right]$ [28], where $P_{c}$ is the SG-standardized urinary metabolite concentration $(\mu \mathrm{g} / \mathrm{L}), P$ is the concentration of the metabolite quantified in the urine sample $(\mu \mathrm{g} / \mathrm{L}), S G_{r e f}$ is the median SG within the study population at each visit $(12$ weeks $=1.016$, 28 weeks $=1.020,35$ weeks $=1.016$ ), and $S G$ is the measured SG in each sample. For pooled samples $S G_{\text {ref }}$ was the mean SG for all samples (1.017) and SG was the average SG across samples contributing to each individual's pooled sample. For regression analyses, a $\log (2)$-transformation was applied to urinary metabolite concentrations to decrease the influence of extreme values on effect estimates.

\section{Sociodemographic and dietary predictors of urinary flame retardant metabolite concentrations}

At enrollment women completed a brief questionnaire describing their highest level of education attained and household income. Additional demographic (maternal age and race), anthropometric (weight and height), and perinatal factors (parity) were abstracted from the women's medical records following delivery. Pre-pregnancy weight was available in the medical records of most women $(81 \%)$. When available $(n=8)$, we substituted weight from the earliest prenatal care visit for women missing pre-pregnancy weight (11 gestational weeks on average; range 8-15 weeks), because weight gain in early pregnancy is generally not substantial [29]. Maternal height was available for 95\% of women in the study, and body mass index (BMI) was calculated for women with both weight and height data $\left(\mathrm{kg} / \mathrm{m}^{2}\right)$. At approximately six weeks postpartum (mean $=6.7 \pm 1.2$, range $=4.4-9.1$ ) women provided information about their food intake during pregnancy by completing the PrimeScreen, a brief dietary survey with good validity and reproducibility compared to more extensive semi-quantitative food frequency questionnaires [30].

\section{Statistical analysis}

We examined the distributions and frequencies of participants' sociodemographic characteristics. For flame retardant metabolites with $>70 \%$ of concentrations above the LOD, we imputed concentrations < LOD with the $\mathrm{LOD} / \sqrt{ } 2[31,32]$. Urinary metabolites that were not frequently detected ( $<70 \%$ detected) were not explored further (Additional file 1: Table S1). For metabolites detected in $>70 \%$ of the individual samples, we report the distribution of SG-standardized concentrations in individual and pooled urine samples. Among women contributing at least two urine samples during pregnancy, we calculated intraclass correlation coefficients (ICCs) using linear mixed models with random intercepts and an unstructured covariance matrix to estimate betweenand within-subject variability of $\log (2)$-transformed urinary flame retardant metabolite concentrations over the 
course of pregnancy. In order to further examine changes in urinary flame retardant metabolite concentrations over the course of pregnancy, we assessed the association between repeated measurements of urinary metabolites and gestational week of urine collection using linear mixed models with unstructured covariance. We explored whether the concentrations of metabolites of interest were associated with time of urine collection (morning versus afternoon) using linear mixed models with unstructured covariance.

In order to assess whether within-subject pooling of urine might be useful for reducing potential exposure misclassification in future epidemiological studies [33], we investigated how well the urinary flame retardant metabolite concentrations measured in the pooled urine samples approximated average urinary metabolite concentrations over the course of pregnancy. We took the average concentration of each metabolite across the individual samples for each woman and calculated the ICCs comparing this arithmetic average with the measured concentration in her pooled urine sample. We also created Bland Altman plots to visualize the agreement between concentrations in pooled samples and the arithmetic average. We additionally calculated Spearman correlations among the frequently detected urinary flame retardant metabolites in the pooled samples.

We created linear mixed models with unstructured covariance to assess the association of each individual sociodemographic predictor of interest with repeated measurements of $\log (2)$-transformed SG-standardized flame retardant metabolite concentrations; models were adjusted for gestational week of urine collection. Predictors of interest included: maternal age, pre-pregnancy weight, pre-pregnancy BMI, maternal race, education, household income, and parity. We also fit multivariable linear mixed models with unstructured covariance matrices for each PFR metabolite to estimate associations of repeated $\log (2)$-transformed, SG-standardized, urinary concentrations with continuous age at delivery (years), pre-pregnancy weight $(\mathrm{kg})$, household income (dollars), gestational week of urine collection (weeks), and indicator variables for race/ethnicity, education, and parity in the multivariable models. To further assess the utility of pooled urine samples, we created comparable multivariable linear regression models to assess the association of concentrations of PFR metabolites in the pooled urine sample with sociodemographic predictors for comparison with the results of the linear mixed models.

Multivariable linear mixed models with unstructured covariance matrices were employed to examine dietary predictors of urinary flame retardant metabolites. Each model described the association of a single dietary predictor of interest with repeated measures of $\log (2)$ transformed SG-standardized metabolite concentrations, adjusted for continuous age at delivery (years), pre-pregnancy weight $(\mathrm{kg})$, household income (dollars), gestational week at urine collection (weeks), and indicator variables for race/ethnicity, education, and parity. We included observations from 51 women with complete covariate data in the final multivariable models. For all regression models described above, we estimated percent difference in metabolite concentration $\left[\%\right.$ diff $\left.=\left(2^{\beta}-1\right)^{*} 100\right]$, where $\beta$ is the estimated regression coefficient of interest.

\section{Results}

On average, women in our study were 29.5 years old at delivery, weighed $74.7 \mathrm{~kg}$ ( 165 pounds) prior to pregnancy, and had a pre-pregnancy BMI of $27.7 \mathrm{~kg} / \mathrm{m}^{2}$. Most participants were non-Hispanic white (59\%), had a bachelor's, graduate, or other professional degree (44\%), and were parous (54\%) (Table 1).

We frequently detected (\% detected): DPhP (95\%), BDCPP (93\%), and BCEP (74\%), and only occasionally BCPP (53\%), DBuP (33\%), DpCP (18\%), and DoCP (1\%). DBzP and TBBA were not detected in any of the urine samples (Additional file 1: Table S1). Median (IQR) BCEP, BDCPP, and DPhP, SG-standardized concentrations from pooled samples were: $0.31 \mu \mathrm{g} / \mathrm{L}$ (0.17-0.54), $1.18 \mu \mathrm{g} / \mathrm{L}(0.64-2.19)$, and $0.93 \mu \mathrm{g} / \mathrm{L}$ (0.72-1.97) (Additional file 1: Table S3).

Applying the ICC metrics of Rosner (ICCs $\leq 0.4$, between 0.4 and 0.75 , and $\geq 0.75$ designate poor, fair to good, and excellent reproducibility, respectively) [34], we observed fair to good reproducibility in BCEP (ICC = 0.50; 95\% CI: 0.37, 0.58), BDCPP (ICC $=0.60 ; 95 \% \mathrm{CI}$ : $0.54,0.66$ ), and DPhP (ICC $=0.43 ; 95 \%$ CI: $0.36,0.50$ ) (Fig. 1; Additional file 1: Table S4). Each additional gestational week at urine collection was marginally associated with 1.4\% higher urinary BCEP (95\% CI:-0.3, 3.1), $1.2 \%$ higher BDCPP (95\% CI: 0.0, 2.4), and 0.9\% higher DPhP (95\% CI: -0.1, 1.9) (Table 2). The majority of urine samples $(74 \%)$ were collected before noon. No statistically significant differences in concentrations between samples collected in the morning and afternoon were observed. However, BCEP concentrations were 44\% higher in samples collected during the afternoon, though the confidence interval was wide (95\% CI: $-10,131)$. We observed excellent agreement between the average urinary flame retardant metabolite concentrations across pregnancy samples versus concentrations in the pooled urine samples; ICC (95\% CI): BCEP $=0.95(0.91,0.97)$, $\mathrm{BDCPP}=0.89(0.86,0.95)$, and $\mathrm{DPhP}=0.93(0.92,0.95)$ (Additional file 1: Table S5). The Bland Altman plots also supported high agreement between methods (Additional file 1: Figure S1). The SG-standardized 
Table 1 Selected participant characteristics and unadjusted percent difference in urinary flame retardant metabolite concentrations as a function of sociodemographic predictors

\begin{tabular}{|c|c|c|c|c|c|}
\hline \multirow[b]{3}{*}{ Characteristics } & \multirow[t]{3}{*}{$\mathrm{n}$} & \multirow{3}{*}{$\begin{array}{l}\text { Mean } \pm \text { SD } \\
(\%)\end{array}$} & \multicolumn{3}{|c|}{ Urinary flame retardant metabolites during pregnancy ${ }^{a}$} \\
\hline & & & \multirow{2}{*}{$\begin{array}{l}\text { BCEP } \\
\% \text { diff }(95 \% \mathrm{Cl})\end{array}$} & \multirow{2}{*}{$\begin{array}{l}\text { BDCPP } \\
\% \text { diff }(95 \% \mathrm{Cl})\end{array}$} & \multirow{2}{*}{$\begin{array}{l}\text { DPhP } \\
\% \text { diff }(95 \% \mathrm{Cl})\end{array}$} \\
\hline & & & & & \\
\hline Maternal Age (years) & 52 & $29.5 \pm 4.5$ & $-3.2(-10.2,4.2)$ & $-4.9(-9.7,0.1)^{*}$ & $-1.4(-5.2,2.5)$ \\
\hline Maternal Weight (kg) & 56 & $74.7 \pm 20.0$ & $0.9(0.0,1.9)^{*}$ & $1.3(0.1,2.5)^{* *}$ & $0.5(-0.1,1.1)$ \\
\hline Maternal BMI $\left(\mathrm{kg} / \mathrm{m}^{2}\right)$ & 56 & $27.7 \pm 6.8$ & $1.7(-1.5,5.0)$ & $3.5(0.2,6.9)^{* *}$ & $1.5(-0.2,3.3)^{*}$ \\
\hline \multicolumn{6}{|l|}{ Maternal Race ${ }^{b}$} \\
\hline Non-Hispanic White & 35 & (59) & 0 (reference) & 0 (reference) & 0 (reference) \\
\hline Other & 20 & (34) & $9.1(-34.4,81.2)$ & $40.5(-10.2,119.9)$ & $10(-20.7,52.6)$ \\
\hline \multicolumn{6}{|l|}{ Maternal Education ${ }^{c}$} \\
\hline High School or Less & 16 & (27) & 0 (reference) & 0 (reference) & 0 (reference) \\
\hline Tech school/Some College & 16 & (27) & $1.3(-53.2,119.6)$ & $-40.7(-65.7,2.8)^{*}$ & $-1.1(-35.4,51.6)$ \\
\hline \multirow[t]{2}{*}{ Bachelor's/Graduate/Professional } & 26 & (44) & $-30.3(-68.2,52.9)$ & $-47.1(-67.8,-13.3)^{* *}$ & $-4.0(-36.2,44.4)$ \\
\hline & & & p-trend $=0.30$ & p-trend $=0.02$ & p-trend $=0.83$ \\
\hline \multicolumn{6}{|l|}{ Household Income } \\
\hline$<\$ 25,000$ & 20 & (34) & 0 (reference) & 0 (reference) & 0 (reference) \\
\hline$\$ 25,000-100,000$ & 20 & (34) & $-6.5(-51.2,79.2)$ & $-41.3(-64.2,-3.8)^{* *}$ & $-6.3(-35.1,35.4)$ \\
\hline \multirow[t]{2}{*}{$>\$ 100,000$} & 18 & (31) & $-29.9(-63.1,33.3)$ & $-42.8(-64.6,-7.6)^{* *}$ & $-10.2(-35.8,25.6)$ \\
\hline & & & p-trend $=0.27$ & p-trend $=0.02$ & p-trend $=0.52$ \\
\hline \multicolumn{6}{|l|}{ Parity $^{d}$} \\
\hline Nulliparous & 23 & (39) & 0 (reference) & 0 (reference) & 0 (reference) \\
\hline Parous & 32 & (54) & $-1.0(-41.5,67.7)$ & $31.2(-16.6,106.3)$ & $1.6(-26.3,40.0)$ \\
\hline
\end{tabular}

BMI Body Mass Index, SD Standard deviation, ${ }^{*} p<0.10,{ }^{* *} p<0.05$

a Percent difference calculated from linear mixed models using the individual sociodemographic factor of interest to predict repeated measurements at 12,28 and 35 weeks' gestation of $\log (2)$-transformed specific gravity standardized concentration of each urinary flame retardant metabolite adjusted for gestational week of urine sample

${ }^{b}$ Missing $n=4$

c Missing $n=1$

${ }^{\mathrm{d}}$ Missing $\mathrm{n}=4$

urinary concentrations of BCEP, BDCPP, and $\mathrm{DPhP}$ from pooled samples were moderately positively correlated to one another with Spearman correlation coefficients ranging from 0.46 to 0.51 ( $p$-values $<0.01$ ) (Additional file 1: Table S6).

In the regression models assessing individual sociodemographic predictors adjusted for gestational week of urine collection, each 1 year increase in maternal age at delivery was marginally associated with $4.9 \%$ lower urinary BDCPP (95\% CI: -9.7, 0.1). Each $1 \mathrm{~kg}$ increase in pre-pregnancy weight was suggestively associated with $0.9 \%$ higher BCEP $(95 \% \mathrm{CI}: 0.0,1.9)$ and significantly associated with $1.3 \%$ higher BDCPP (95\% CI: 0.1, 2.5) (Additional file 1: Figure S3); each $1 \mathrm{~kg} / \mathrm{m}^{2}$ increase in pre-pregnancy BMI was associated with statistically significantly higher urinary BDCPP $(3.5 \%$; $95 \%$ CI: $0.2,6.9)$, and suggestively associated with higher DPhP (1.5\%; $95 \%$ CI: $-0.2,3.3)$. Women with more education tended to have lower BDCPP concentrations (p-trend 0.02). Likewise, women with higher household income had lower urinary concentrations of BDCPP (p-trend 0.02) (Table 1). In the regression models which simultaneously included all sociodemographic factors of interest, each $1 \mathrm{~kg}$ increase in pre-pregnancy weight was associated with a statistically significant increases in BCEP (1.1\%, 95\% CI: $0.1,2.1)$ and BDCPP $(1.5 \%, 95 \%$ CI: $0.3,2.7)$, and marginally higher DPhP $(0.5 \%, 95 \%$ CI: $0.0,1.1)$. Parous women had $64.1 \%$ greater urinary BDCPP than nulliparous women (95\% CI: 5.2, 155.9) (Table 2). Similar patterns of results were obtained from exploratory multivariable models including BMI rather than weight (Additional file 1: Table S7). Maternal weight remained a suggestive predictor of BCEP $(1.6 \%$; 95\% CI: $0.0,3.3)$ and BDCPP $(1.2 \%$; $95 \%$ CI: $0.1,2.3$ ) in the pooled urine samples; whereas, parity was of comparable magnitude but no longer a statistically significant predictor of urinary BDCPP concentration in pooled urine samples (Additional file 1: Table S8).

Overall, dietary factors were not predictive of greater urinary flame retardant metabolite concentrations in urine during pregnancy in multivariable regression models 


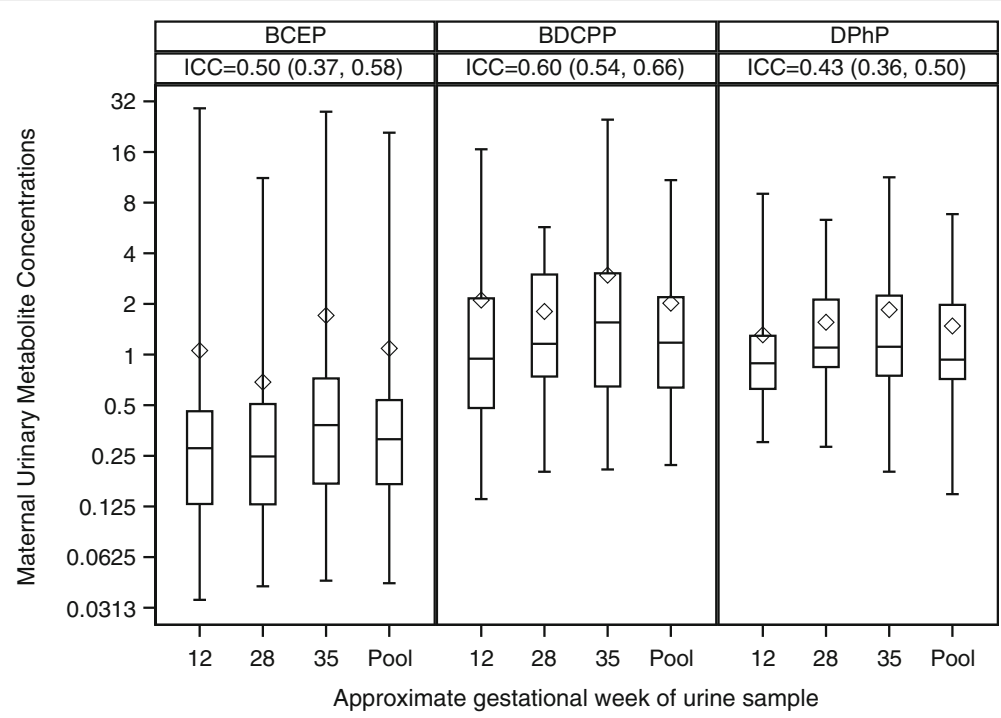

Fig. 1 Distributions of specific gravity standardized maternal urinary replacement flame retardant metabolite concentrations (in $\mu \mathrm{g} / \mathrm{L}$ ) in urine samples from 12, 28, and 35 gestational weeks and the pooled urine sample. Intraclass correlation coefficients (ICCs) and 95\% confidence intervals for the 12,28 , and 35 week samples were $B C E P=0.50, B D C P P=0.60$, and $\mathrm{DPhP}=0.43$

adjusted for relevant sociodemographic characteristics (Additional file 1: Figure S2 and Table S9). Lower urinary BCEP was associated with more frequent consumption of meat (beef/pork/lamb) in a main dish $(-26.1 \%, 95 \% \mathrm{CI}$ : $-43.8,-2.8)$. Lower urinary BDCPP concentrations were observed among women who more frequently consumed leafy green vegetables (including spinach, lettuce, kale, turnip greens, and bok choy) $(-19.9 \%, 95 \%$ CI: -33.5 , $-3.5)$ or carrots $(-25.9 \%, 95 \%$ CI: $-40.7,-7.4)$. More frequent consumption of citrus fruits was associated with lower urinary DPhP concentrations $(-20.6 \%, 95 \%$ CI: $-28.5,-11.8$ ).

\section{Discussion}

In this pilot study, we investigated patterns and predictors of urinary PFR metabolite concentrations over the course of pregnancy and assessed potential sociodemographic and dietary predictors. We found that BCEP, $\mathrm{BDCPP}$, and $\mathrm{DPhP}$ were frequently detected in the urine of pregnant women in our study. Concentrations of these metabolites had fair to good reproducibility over the course of pregnancy. Weight and BMI were positively associated with flame retardant metabolite concentrations in urine in this cohort. Suggestive inverse associations between age, education, and household income with BDCPP were observed, and parous women had statistically significantly higher BDCPP urinary concentrations than nulliparous women. Overall, we did not observe strong associations between dietary predictors and urinary BCEP, BDCPP, or DPhP.

Both BDCPP and DPhP have been frequently detected in the urine of mothers $[13,14,24,25]$, young children
$[12,13,24]$, and the general population [10-12, 15-18]. Urinary concentrations of BDCPP and DPhP observed in our study were generally consistent with those previously observed among pregnant women [14, 25] (Additional file 1: Table S10). To the best of our knowledge, only one previous study $(n=16)$ reported urinary concentrations of BCEP among members of the general population in the United States; median concentrations observed in this pilot study $(0.31 \mu \mathrm{g} / \mathrm{L})$ were lower than in the prior study $(0.63 \mu \mathrm{g} / \mathrm{L})$ (Additional file 1 : Table S10). Although only three of the metabolites were detected in $>70 \%$ of samples, we measured a total of nine. The LODs for the other six compounds were quite comparable to the LODs of the three frequently detected flame retardant metabolites, suggesting that exposure to the precursors of these six metabolites may be limited among women in our study.

Our findings concerning temporal variability of $\mathrm{BDCPP}$ and $\mathrm{DPhP}$ are quite consistent with previous research. Hoffman et al. assessed urinary BDCPP and $\mathrm{DPhP}$ among eight pregnant women in North Carolina at two time points during pregnancy (18 and 28 weeks gestation) and following delivery, and reported fair to good reproducibility of both BDCPP (ICC $=0.4 ; 95 \%$ CI $0.2,0.6)$ and DPhP (ICC $=0.4 ; 95 \%$ CI $0.2,0.6)$ [14]. Likewise, Hoffman et al. observed excellent agreement of BDCPP (ICC $=0.81 ; 95 \%$ CI 0.75 , 0.86 ) and fair agreement of DPhP (ICC $=0.51 ; 95 \%$ CI:0.42, 0.63) concentrations across 49 total repeated urine samples collected over a 5 day period from 11 healthy adults [8]. Meeker et al. [11] assessed urinary BDCPP and DPhP concentration among seven men in 
Table 2 Adjusted percent difference in urinary flame retardant metabolite concentrations as a function of sociodemographic predictors

\begin{tabular}{|c|c|c|c|}
\hline \multirow[b]{3}{*}{ Predictors } & \multicolumn{3}{|c|}{ Urinary flame retardant metabolite during pregnancy ${ }^{a}$} \\
\hline & BCEP & BDCPP & $\mathrm{DPhP}$ \\
\hline & $\%$ diff $(95 \% \mathrm{Cl})$ & $\%$ diff $(95 \% \mathrm{Cl})$ & $\%$ diff $(95 \% \mathrm{Cl})$ \\
\hline Maternal Age & $-0.7(-10,9.6)$ & $-3.8(-10.9,3.9)$ & $-2.2(-6.9,2.8)$ \\
\hline Maternal Weight (kg) & $1.1(0.1,2.1)^{* *}$ & $1.5(0.3,2.7)^{* *}$ & $0.5(0.0,1.1)^{*}$ \\
\hline \multicolumn{4}{|l|}{ Maternal Race } \\
\hline Non-Hispanic White & 0 (reference) & 0 (reference) & 0 (reference) \\
\hline Other & $-23.4(-67.4,80)$ & $18.1(-31.6,103.8)$ & $10.4(-31.5,77.9)$ \\
\hline \multicolumn{4}{|l|}{ Maternal Education } \\
\hline High School or Less & 0 (reference) & 0 (reference) & 0 (reference) \\
\hline Tech school/Some College & $3.9(-52.9,129.2)$ & $-40.1(-65.7,4.5)^{*}$ & $-0.3(-36.5,56.6)$ \\
\hline \multirow[t]{2}{*}{ Bachelor's or more } & $-29.6(-76.1,107.3)$ & $-43.7(-75.5,29.7)$ & $8.7(-33.0,76.5)$ \\
\hline & $p$-trend $=0.57$ & $p$-trend $=0.14$ & $p$-trend $=0.74$ \\
\hline Household Income $e^{b}$ & $-0.9(-8.0,6.6)$ & $3.4(-4.8,12.2)$ & $1.1(-3.6,6.0)$ \\
\hline \multicolumn{4}{|l|}{ Parity } \\
\hline Nulliparous & 0 (reference) & 0 (reference) & 0 (reference) \\
\hline Parous & $-1.7(-42.6,68.6)$ & $64.1(5.2,155.9)^{* *}$ & $13.6(-21.7,64.6)$ \\
\hline Gestational week $^{c}$ & $1.4(-0.3,3.1)^{*}$ & $1.2(0.0,2.4)^{*}$ & $0.9(-0.1,1.9)^{*}$ \\
\hline
\end{tabular}

Massachusetts at nine time points over the course of a three month period, and reported fair to good reproducibility of urinary BDCPP ( $\mathrm{CCC}=0.55 ; 95 \%$ CI $0.31,0.77$ ) and poor reproducibility of DPhP $(\mathrm{ICC}=0.35 ; 95 \%$ CI $0.17,0.59$ ). However, a study of 51 office workers in Massachusetts observed poor agreement across three measurements of urinary DPhP taken over the course of $\sim 12$ months (ICC $=0.13 ; 95 \%$ CI 0.02, 0.52) [19], suggesting that variability of DPhP may be greater over longer periods of observation. Our results are consistent with fair to good reproducibility of BDCPP and DPhP over the course of about six months and add information suggesting that urinary BCEP concentrations have similar variability to BDCPP and DPhP among pregnant women. In our adjusted models, we observed marginally statistically significant positive associations of gestational week of urine collection with concentrations of BCEP, BDCPP, and DPhP; whereas, Hoffman et al. observed a statistically imprecise but inverse association between gestational week of urine collection with urinary BDCPP and DPhP [25]. Although the studies conflict in terms of the direction of the association, both studies highlight the need to account for gestational week of urine collection in future epidemiologic research, particularly in studies that rely on only one urine sample from pregnancy for exposure measurement.

We observed good agreement between concentrations of BCEP, BDCPP, and DPhP measured in pooled samples from individual women and the average of concentrations from each woman's serial urine samples. Evidence from animal studies [35-37] and human studies [38, 39] suggests that the half-lives of PFRs in the body are likely to be $<24 \mathrm{~h}$. In situations in which humans are exposed via multiple sources to a chemical with a short half-life in the body, exposure assessment with a single urine sample may lead to exposure misclassification and within-subject pooling of urine samples represents an efficient and cost-effective alternative to averaging the concentrations in serial samples [33]. Overall, the sociodemographic predictors of urinary PFR concentrations identified by the linear mixed models taking advantage of repeated measurements were similar to the multivariable linear regression using only the pooled sample. While we were able to collect up to 3 samples per woman over the course of pregnancy, additional samples may be necessary to realize the full potential of pooled samples to prevent exposure misclassification, but the exact amount needed will depend on the reproducibility of the repeated samples [33]. Our findings suggest 
that urinary flame retardant metabolites are potentially good candidates for use of within-subject pooling in future etiologic studies of the health outcomes associated with exposure to PFRs. Additionally, future studies could collect multiple urine samples during etiologically relevant periods (e.g., first trimester) and use pooling to provide a more efficient and costeffective estimation of exposure during specific exposure windows of interest.

Prior examination of predictors of urinary BCEP, BDCPP, and DPhP among adults has been somewhat limited. Our findings generally suggest that women with higher weight or greater BMI tend to have higher concentrations of BCEP, BDCPP, and DPhP in their urine than lighter women. This is consistent with one prior study of pregnant women in which women who were overweight or obese before pregnancy tended to have higher urinary concentrations of BDCPP and DPhP than women with normal pre-pregnancy body mass index [25]. One possible explanation is that TPhP, the parent PFR of DPhP, has been used as a plasticizer (potentially as a substitute for dibutyl phthalates) in nail polish [38], and may be used as a plasticizer or phthalate substitute in other personal care products. If the relevant plasticizers are present in personal care products, this is one possible, though speculative, explanation for observing greater concentrations of urinary BCEP, BDCPP, and DPhP among heavier women, who may have greater body surface area and thus greater potential for exposure to chemicals present in personal care products. Further studies could examine use of personal care products to explore this hypothesis. Although we were unable to identify dietary predictors of PFRs in the present study, higher urinary concentrations of PFR metabolites among heavier women could also be explained by as yet undefined sources of PFR exposure in food or food packaging.

We observed a suggestive but not statistically significant inverse association between age and urinary BDCPP in the model assessing the individual influence of age; however age was not an important predictor of BDCPP in our multivariable models. Three prior studies suggest that age is inversely associated with urinary concentrations of BDCPP $[8,10,17]$. However, one larger study conducted by Hoffman et al. among pregnant women saw no association of BDCPP or DPhP with maternal age, race, or education [25]. We likewise did not observe differences in BDCPP or DPhP according to race/ethnicity, but saw some suggestion that women with higher educational attainment had lower urinary BDCPP concentrations. Sociodemographic predictors such as income, education, and race have frequently been associated with exposures to some environmental endocrine disrupting chemicals [40-43].

We speculate that the observed association of greater urinary BDCPP among parous women is at least potentially related to a higher prevalence of foam-containing infant and childhood products in the homes of pregnant women with previous children, as substantial evidence exists suggesting that PFRs are commonly found in a wide variety of foam-containing infant and baby products $[2,9,12]$. Additional research would be useful to confirm this hypothesis. In contrast, Hoffman et al. observed similar urinary BDCPP concentrations among parous and primiparous women, but urinary $\mathrm{DPhP}$ concentrations were elevated among parous women in their study population [25]. Collectively, our findings suggest that future etiologic studies assessing health outcomes potentially associated with exposure to TCEP, TDCPP, and TPhP would benefit from considering anthropometrics (i.e., weight), sociodemographic, and perinatal factors that may be associated with exposure.

We did not observe clear patterns between dietary factors and PFR metabolites in urine. PBDE flame retardants have commonly been found in meat, eggs, fish and seafood, animal fats and vegetable oils, milk and dairy products, and bakery products $[44,45]$. Thus, the lower urinary $\mathrm{BCEP}$ concentrations among individuals more frequently consuming meat was unexpected. However, the persistence and bioaccumulative properties of PFRs are expected to differ from those of PBDEs [46]. Cequier et al. observed greater urinary BDCPP concentrations among children consuming more sugar and mothers consuming more cakes during the preceding $24 \mathrm{~h} \mathrm{[23].}$ The observed pattern of results in the present study may tentatively suggest that individuals eating more vegetables and fruits tend to have lower concentrations of urinary PFRs (Additional file 1: Figure S2). However, a more detailed assessment of diet, as well as direct analysis of food and food packaging to assess the presence of PFRs, may be necessary to further elucidate potential dietary sources of PFR exposure and are beyond the scope of the present pilot.

Our study has several limitations and strengths worth noting. Although this pilot study was small in size, we demonstrated that it is feasible to collect multiple urine samples over the course of pregnancy to better quantify gestational exposure to PFRs and create pooled urine samples that have excellent agreement with the average of individual measurements. While we did not directly measure SG in the pooled urine samples, the close agreement of ICCs using values that were and were not standardized for SG suggests that this was not a source of bias in our reported ICCs. We had a somewhat limited collection of covariates, but we were able to provide additional insight into several common sociodemographic factors known to influence exposure to other endocrine disrupting chemicals. Likewise, the use of an abbreviated dietary questionnaire may have prevented identification of potential dietary predictors of urinary 
PFRs. However, we were able to provide preliminary data to suggest that diet may not be the predominant source of exposure to PFRs. We were not able to assess pre- or post-pregnancy concentrations of urinary PFRs, thus our findings may not be generalizable to non-pregnant populations. Future research could incorporate preconception measurement of PFRs in order to better understand how the normal physiologic changes of pregnancy may influence urinary PFR concentrations.

\section{Conclusions}

Collectively, our findings suggest that pregnant women in the United States are commonly exposed to TCEP, TDCPP, and TPhP. Urinary concentrations of BCEP, BDCPP, and DPhP are moderately variable over the course of pregnancy, such that future research may benefit from the use of within-person pooling of urine samples to reduce potential exposure misclassification. Although we did not observe clear evidence of dietary predictors of urinary PFR metabolites, future research would benefit from examining additional or more detailed potential food sources of PFRs, especially because body size was a predictor of urinary PFR metabolite concentrations in this and other cohorts. Considering the presence of these chemicals in the urine of pregnant women, increasing our understanding of possible health effects associated with exposure to PFRs during the sensitive window of pregnancy is of public health interest.

\section{Additional file}

Additional file 1: Table S1. Parent flame retardants and limits of detection and percent detected of associated urinary metabolites. Table S2. Urinary concentrations of flame retardant metabolites and coefficients of variation (CV) in 12 blinded replicates. Table S3. Medians and selected percentiles of urinary flame retardant metabolites in urine during pregnancy. Table S4. Intraclass correlation coefficients of pilot study participants' urinary flame retardant metabolite concentrations during pregnancy. Table S5. Intraclass correlation coefficients of average urinary flame retardant metabolite concentrations during pregnancy across pregnancy samples versus concentrations from a pooled urine sample among pilot study participants. Table S6. Spearman correlations among specific gravity standardized concentrations of flame retardant metabolites from the pooled urine samples. Table S7. Adjusted percent difference in urinary flame retardant metabolite concentrations as a function of sociodemographic predictors. Table S8. Adjusted percent difference in urinary flame retardant metabolite concentrations in pooled urine samples as a function of sociodemographic predictors $(n=51)$. Table S9. Dietary predictors of urinary flame retardant metabolites during pregnancy. Table S10. Central tendency of urinary flame retardant metabolites $(\mathrm{ng} / \mathrm{mL})$ among adults in the United States. Figure S1. Bland Altman plots of the difference between urinary flame retardant metabolite concentrations measured in pooled urine samples and the average of concentrations from urine samples collected across pregnancy versus the mean of the two measurements. Figure S2. Adjusted percent difference in urinary flame retardant metabolites and 95\% confidence intervals as a function of dietary factors. Figure S3. Scatterplots and trend lines for of log(2)transformed urinary flame retardant concentrations from pooled urine samples by maternal weight (kg). (DOCX $452 \mathrm{~kb}$ )

\section{Abbreviations}

BCEP: Bis-2-chloroethyl phosphate; BCPP: Bis-(1-chloro-2-propyl) phosphate: BDCPP: Bis(1,3-dichloro-2-propyl) phosphate; BMI: Body mass index; CDC: Centers for disease control and prevention; DBuP: Dibutyl phosphate; DBzP: Di-benzyl-phosphate; DoCP: Di-o-cresylphosphate; DpCP: Di-pcresylphosphate; DPhP: Diphenyl phosphate; ICC: Intraclass correlation coefficient; IQR: Interquartile range; LOD: Limit of detection;

PFR: Organophosphate flame retardant; QC: Quality control; SG: Specific gravity; TBBA: 2,3,4,5-tetrabromobenzoic acid; TBuP: Tributyl phosphate; TCEP: Tris (2-chloroethyl) phosphate; TDCPP: Tris (1,3-dichloro-2-propyl) phosphate; TPhP: Triphenyl phosphate; US: United States; WIHRI: Women \& Infants Hospital of Rhode Island

\section{Acknowledgements}

The authors acknowledge Paula Restrepo and LaTasha Williams at the Centers for Disease Control and Prevention laboratory who performed the measurements of PFRs.

\section{Funding}

This research was supported by Research Seed Funding from the Office of the Vice President for Research at Brown University (Providence, RI) and NIEHS grants R01-ES024381 and R00-ES020346. Dr. Romano was supported by NIGMS grant P20-GM104416.

\section{Availability of data and materials}

The datasets used and/or analyzed during the current study are available from the corresponding author on reasonable request.

\section{Authors' contributions}

$M R, N H, K K, S M, M P, D S, A C, E W$, and JB designed the study and critically reviewed and edited the manuscript. MR completed the majority of the statistical analyses and drafted the initial manuscript. ME provided statistical support, created the figures, and critically edited the methods portion of the manuscript. AC and NJ quantified the urinary PFRs and drafted the associated methods for the manuscript. EW and JB supervised data collection for the study and provided critical feedback on the analysis plan and manuscript. All authors read and approved the final manuscript.

\section{Competing interests}

The authors declare that they have no competing interests.

\section{Consent for publication}

$\mathrm{n} / \mathrm{a}$.

\section{Ethics approval and consent to participate}

Prior to engaging in study activities all participants provided written informed consent. All protocols were approved by the WIHRI institutional review board, and the involvement of the CDC laboratory did not constitute engagement in human subjects research.

\section{Disclaimer}

The findings and conclusions in this report are those of the authors and do not necessarily represent the official position of the Centers for Disease Control and Prevention (CDC). Use of trade names is for identification only and does not imply endorsement by the CDC, the Public Health Service, or the US Department of Health and Human Services.

\section{Publisher's Note}

Springer Nature remains neutral with regard to jurisdictional claims in published maps and institutional affiliations.

\section{Author details}

${ }^{1}$ Department of Epidemiology, Brown University School of Public Health, Providence, RI, USA. ²Department of Epidemiology, Geisel School of Medicine at Dartmouth, Lebanon, NH, USA. ${ }^{3}$ Department of Epidemiology (Chronic Diseases), Yale University School of Public Health, New Haven, CT, USA. ${ }^{4}$ Division of Laboratory Sciences, National Center for Environmental Health, Centers for Disease Control and Prevention, Atlanta, GA, USA.

${ }^{5}$ Department of Pathology and Laboratory Medicine, Warren Alpert Medical School of Brown University, Providence, RI, USA. ${ }^{6}$ Department of Anthropology, Brown University, Providence, RI, USA. ${ }^{7}$ Department of 
Obstetrics and Gynecology, Warren Alpert Medical School of Brown University, and Women \& Infants Hospital of Rhode Island, Providence, Rl, USA.

\section{Received: 4 November 2016 Accepted: 30 March 2017} Published online: 11 April 2017

\section{References}

1. Stapleton HM, Sharma S, Getzinger G, Ferguson PL, Gabriel M, Webster TF, Blum A. Novel and high volume use flame retardants in US couches reflective of the 2005 PentaBDE phase out. Environ Sci Technol. 2012;46(24): 13432-9.

2. Stapleton HM, Klosterhaus S, Keller A, Ferguson PL, van Bergen S, Cooper E, Webster TF, Blum A. Identification of flame retardants in polyurethane foam collected from baby products. Environ Sci Technol. 2011:45(12):5323-31.

3. Jayatilaka NK, Restrepo P, Williams L, Ospina M, Valentin-Blasini L, Calafat AM. Quantification of three chlorinated dialkyl phosphates, diphenyl phosphate, 2,3,4,5-tetrabromobenzoic acid, and four other organophosphates in human urine by solid phase extraction-high performance liquid chromatography-tandem mass spectrometry. Anal Bioanal Chem. 2017:409(5):1323-32.

4. van der Veen I, de Boer J. Phosphorus flame retardants: properties, production, environmental occurrence, toxicity and analysis. Chemosphere. 2012:88(10):1119-53.

5. Andresen JA, Grundmann A, Bester K. Organophosphorus flame retardants and plasticisers in surface waters. Sci Total Environ. 2004:332(1-3):155-66.

6. Covaci A, Harrad S, Abdallah MA, Ali N, Law RJ, Herzke D, de Wit CA. Nove brominated flame retardants: a review of their analysis, environmental fate and behaviour. Environ Int. 2011;37(2):532-56.

7. Solbu K, Thorud S, Hersson M, Ovrebo S, Ellingsen DG, Lundanes E, Molander P. Determination of airborne trialkyl and triaryl organophosphates originating from hydraulic fluids by gas chromatography-mass spectrometry. Development of methodology for combined aerosol and vapor sampling. J Chromatogr A. 2007;1161(1-2):275-83.

8. Hoffman K, Garantziotis S, Birnbaum LS, Stapleton HM. Monitoring indoor exposure to organophosphate flame retardants: hand wipes and house dust. Environ Health Perspect. 2015:123(2):160-5.

9. Fang M, Webster TF, Gooden D, Cooper EM, McClean MD, Carignan C, Makey C, Stapleton HM. Investigating a novel flame retardant known as V6: measurements in baby products, house dust, and car dust. Environ Sci Technol. 2013;47(9):4449-54.

10. Carignan CC, McClean MD, Cooper EM, Watkins DJ, Fraser AJ, HeigerBernays W, Stapleton HM, Webster TF. Predictors of tris(1,3-dichloro-2 propyl) phosphate metabolite in the urine of office workers. Environ Int. 2013;55:56-61.

11. Meeker JD, Cooper EM, Stapleton HM, Hauser R. Urinary metabolites of organophosphate flame retardants: temporal variability and correlations with house dust concentrations. Environ Health Perspect. 2013;121(5):580-5.

12. Hoffman K, Butt CM, Chen A, Limkakeng Jr AT, Stapleton HM. High exposure to organophosphate flame retardants in infants: associations with baby products. Environ Sci Technol. 2015;49(24):14554-9.

13. Butt CM, Congleton J, Hoffman K, Fang M, Stapleton HM. Metabolites of organophosphate flame retardants and 2-ethylhexyl tetrabromobenzoate in urine from paired mothers and toddlers. Environ Sci Technol. 2014;48(17): 10432-8.

14. Hoffman K, Daniels JL, Stapleton HM. Urinary metabolites of organophosphate flame retardants and their variability in pregnant women. Environ Int. 2014;63:169-72.

15. Cooper EM, Covaci A, van Nuijs AL, Webster TF, Stapleton HM. Analysis of the flame retardant metabolites bis(1,3-dichloro-2-propyl) phosphate (BDCPP) and diphenyl phosphate (DPP) in urine using liquid chromatography-tandem mass spectrometry. Anal Bioanal Chem. 2011; 401(7):2123-32

16. Dodson RE, Van den Eede N, Covaci A, Perovich $\amalg$, Brody JG, Rudel RA. Urinary biomonitoring of phosphate flame retardants: levels in California adults and recommendations for future studies. Environ Sci Technol. 2014; 48(23):13625-33.

17. Van den Eede N, Heffernan AL, Aylward LL, Hobson P, Neels H, Mueller JF, Covaci A. Age as a determinant of phosphate flame retardant exposure of the Australian population and identification of novel urinary PFR metabolites. Environ Int. 2015;74:1-8.
18. Van den Eede N, Neels H, Jorens PG, Covaci A. Analysis of organophosphate flame retardant diester metabolites in human urine by liquid chromatography electrospray ionisation tandem mass spectrometry. J Chromatogr A. 2013;1303:48-53.

19. Preston EV, McClean MD, Claus Henn B, Stapleton HM, Braverman LE, Pearce EN, Makey CM, Webster TF. Associations between urinary diphenyl phosphate and thyroid function. Environ Int 2017. Epub [February 2, 2017].

20. Meeker JD, Stapleton HM. House dust concentrations of organophosphate flame retardants in relation to hormone levels and semen quality parameters. Environ Health Perspect. 2010;118(3):318-23.

21. Meeker JD, Cooper EM, Stapleton HM, Hauser R. Exploratory analysis of urinary metabolites of phosphorus-containing flame retardants in relation to markers of male reproductive health. Endocr Disruptors (Austin, Tex). 2013; 1(1):e26306

22. State of California Environmental Protection Agency. Proposition 65 List Chemicals known to the state to cause cancer or reproductive toxicity. In: Office of environmental health hazard assessment. 2013.

23. Cequier E, Sakhi AK, Marce RM, Becher G, Thomsen C. Human exposure pathways to organophosphate triesters - a biomonitoring study of motherchild pairs. Environ Intl. 2015;75:159-65.

24. Butt CM, Hoffman K, Chen A, Lorenzo A, Congleton J, Stapleton HM. Regional comparison of organophosphate flame retardant (PFR) urinary metabolites and tetrabromobenzoic acid (TBBA) in mother-toddler pairs from California and New Jersey. Environ Int. 2016;94:627-34.

25. Hoffman K, Lorenzo A, Butt CM, Adair L, Herring AH, Stapleton HM, Daniels $J$. Predictors of urinary flame retardant concentration among pregnant women. Environ Int. 2017;98:96-101.

26. Taylor JK. Quality assurance of chemical measurements. Chelsea: Lewis Publishers; 1987

27. Caudill SP, Schleicher RL, Pirkle JL. Multi-rule quality control for the agerelated eye disease study. Stat Med. 2008;27(20):4094-106.

28. Duty SM, Ackerman RM, Calafat AM, Hauser R. Personal care product use predicts urinary concentrations of some phthalate monoesters. Environ Health Perspect. 2005:113(11):1530-5.

29. Deierlein AL, Siega-Riz AM, Herring A. Dietary energy density but not glycemic load is associated with gestational weight gain. Am J Clin Nutr. 2008;88(3):693-9.

30. Rifas-Shiman SL, Willett WC, Lobb R, Kotch J, Dart C, Gillman MW. Primescreen, a brief dietary screening tool: reproducibility and comparability with both a longer food frequency questionnaire and biomarkers. Public Health Nutr. 2001;4(2):249-54.

31. Glass DC, Gray CN. Estimating mean exposures from censored data: exposure to benzene in the Australian petroleum industry. Ann Occup Hyg. 2001:45(4):275-82.

32. Hornung RW, Reed LD. Estimation of Average Concentration in the Presence of Nondetectable Values. Applied Occupational and Environmental Hygiene. 1990;5(1):46-51.

33. Perrier F, Giorgis-Allemand L, Slama R, Philippat C. Within-subject pooling of biological samples to reduce exposure misclassification in biomarker-based studies. Epidemiology. 2016;27(3):378-88.

34. Rosner B. The intraclass correlation coefficient. In: Fundamentals of biostatistics. Boston: Brooks/Cole, Cengage Learning; 2011.

35. Nomeir AA, Kato S, Matthews HB. The metabolism and disposition of tris(1,3-dichloro-2-propyl) phosphate (Fyrol FR-2) in the rat. Toxicol Appl Pharmacol. 1981:57(3):401-13.

36. Chapman DE, Michener SR, Powis G. Metabolism of the flame retardant plasticizer tris(2-chloroethyl)phosphate by human and rat liver preparations. Fundam Appl Toxicol. 1991;17(2):215-24.

37. Burka LT, Sanders JM, Herr DW, Matthews HB. Metabolism of tris(2chloroethyl) phosphate in rats and mice. Drug Metab Dispos. 1991;19(2): 443-7.

38. Mendelsohn E, Hagopian A, Hoffman K, Butt CM, Lorenzo A, Congleton J, Webster TF, Stapleton HM. Nail polish as a source of exposure to tripheny phosphate. Environ Int. 2016:86:45-51.

39. Carignan CC, Fang M, Stapleton HM, Heiger-Bernays W, McClean MD, Webster TF. Urinary biomarkers of flame retardant exposure among collegiate U.S. gymnasts. Environ Int. 2016;94:362-8.

40. Casas M, Valvi D, Luque N, Ballesteros-Gomez A, Carsin A-E, Fernandez MF, Koch HM, Mendez MA, Sunyer J, Rubio S, et al. Dietary and sociodemographic determinants of bisphenol A urine concentrations in pregnant women and children. Environ Int. 2013;56:10-8. 
41. Sagiv SK, Rifas-Shiman SL, Webster TF, Mora AM, Harris MH, Calafat AM, Ye X, Gillman MW, Oken E. Sociodemographic and perinatal predictors of early pregnancy per- and polyfluoroalkyl substance (PFAS) concentrations. Environ Sci Technol. 2015:49(19):11849-58.

42. Smith KW, Braun JM, Williams PL, Ehrlich S, Correia KF, Calafat AM, Ye X, Ford J, Keller M, Meeker JD, et al. Predictors and variability of urinary paraben concentrations in men and women, including before and during pregnancy. Environ Health Perspect. 2012;120(11):1538-43.

43. James-Todd TM, Chiu Y-H, Zota AR. Racial/ethnic disparities in environmental endocrine disrupting chemicals and women's reproductive health outcomes: epidemiological examples across the life course. Curr Epidemiol Rep. 2016;3(2):161-80.

44. Domingo JL, Marti-Cid R, Castell V, Llobet JM. Human exposure to PBDEs through the diet in Catalonia, Spain: temporal trend. A review of recent literature on dietary PBDE intake. Toxicology. 2008;248(1):25-32.

45. EFSA CONTAM Panel. EFSA panel on contaminants in the food chain: scientific opinion on polybrominated diphenyl ethers (PBDEs) in food. EFSA J. $2011 ; 9(5): 274$

46. Fromme $\mathrm{H}$, Becher $\mathrm{G}$, Hilger B, Volkel W. Brominated flame retardants Exposure and risk assessment for the general population. Int J Hyg Environ Health. 2016;219(1):1-23.

\section{Submit your next manuscript to BioMed Central and we will help you at every step:}

- We accept pre-submission inquiries

- Our selector tool helps you to find the most relevant journal

- We provide round the clock customer support

- Convenient online submission

- Thorough peer review

- Inclusion in PubMed and all major indexing services

- Maximum visibility for your research

Submit your manuscript at www.biomedcentral.com/submit 